\title{
Efficacy of Chemotherapy in Patients with Unresectable or Metastatic Pancreatic Acinar Cell Carcinoma: Potentially Improved Efficacy with Oxaliplatin-Containing Regimen
}

\author{
Changhoon Yoo, MD \\ Bum Jun Kim, MD',2 \\ Kyu-pyo Kim, MD, PhD' \\ Jae-Lyun Lee, MD, PhD' \\ Tae Won Kim, MD, PhD 1 \\ Baek-Yeol Ryoo, MD, PhD \\ Heung-Moon Chang, MD, $\mathrm{PhD}^{1}$
}

${ }^{1}$ Department of Oncology, Asan Medical Center, University of Ulsan College of Medicine, Seoul, ${ }^{2}$ Department of Internal Medicine, Hallym University Medical Center, Hallym University College of Medicine, Seoul, Korea

Correspondence: Heung-Moon Chang, MD, PhD Department of Oncology, Asan Medical Center, University of Ulsan College of Medicine, 88, Olympic-ro, 43-gil, Songpa-gu,

Seoul 05505, Korea

Tel: 82-2-3010-3210

Fax: 82-2-3010-6961

E-mail: changhm@amc.seoul.kr

Received August 12, 2016

Accepted October 18, 2016

Published Online November 9, 2016

${ }^{*}$ Changhoon Yoo and Bum Jun Kim contributed equally to this work.

\begin{abstract}
Purpose
Pancreatic acinar cell carcinoma (ACC) is a rare cancer of the exocrine pancreas. Because of its rare incidence, the efficacy of chemotherapy in this patient population has been largely unknown. Therefore, we retrospectively analyzed the outcomes of patients with advanced pancreatic ACC who received chemotherapy.
\end{abstract}

\section{Materials and Methods}

Between January 1997 and March 2015, 15 patients with unresectable or metastatic pancreatic ACC who received systemic chemotherapy were identified in Asan Medical Center, Korea.

\section{Results}

The median age was 58 years. Eleven and four patients had recurrent/metastatic and locally advanced unresectable disease. The median overall survival in all patients was 20.9 months (95\% confidence interval [Cl], 15.7 to 26.1). As first-line therapy, intravenous 5-fluorouracil were administered in four patients (27\%), gemcitabine in five (33\%), gemcitabine plus capecitabine in two (13\%), oxaliplatin plus 5-fluorouracil/leucovorin (FOLFOX) in two (13\%), and concurrent chemoradiotherapy followed by capecitabine maintenance therapy in two (13\%). The objective response rate (ORR) to chemotherapy alone was $23 \%$ and the median progression-free survival (PFS) was 5.6 months ( $95 \% \mathrm{Cl}, 2.8$ to 8.4). After progression, second-line chemotherapy was administered in eight patients, while four patients received FOLFOX and the other four patients received gemcitabine. The ORR was 38\%, and patients administered FOLFOX had significantly better PFS than those administered gemcitabine (median, 6.5 months vs. 1.4 months; $p=0.007$ ). The ratio of time to tumor progression (TTP) during first-line chemotherapy to TTP at second-line chemotherapy was significantly higher in patients administered FOLFOX (4.07; range, 0.87 to 8.30 ) than in those administered gemcitabine $(0.12$; range, 0.08 to $0.25 ; p=0.029)$.

\section{Conclusion}

Our results suggest that oxaliplatin-containing regimens may have improved activity against pancreatic ACC.

\section{Introduction}

Acinar cell carcinoma (ACC) is a rare pancreatic exocrine malignancy that accounts for $<1 \%$ of all pancreatic neoplasms [1-3]. Because of its rare incidence, with the exception
Key words

Acinar cell carcinoma, Pancreatic neoplasms, Antineoplastic agents, Oxaliplatin 
mon molecular alterations shared with PDAC. Most previous studies have suggested that pancreatic ACC has a better prognosis than PDAC [4,5].

Surgical resection is the only curative treatment modality for localized pancreatic ACC, and patients who received surgery were associated with better survival outcomes [6]. However, approximately half of the patients have metastatic disease at presentation [5,7], and a considerable proportion of patients (57\%-100\%) develop recurrence even after curative surgery $[3,5,8]$. These findings indicate that development of effective systemic chemotherapy is essential for improving survival outcomes in patients with pancreatic ACC.

Despite recent advances in chemotherapy for PDAC after long stagnation, data regarding the chemotherapy for pancreatic ACC remains insufficient, and the most appropriate regimen for first-line chemotherapy is unclear. Moreover, no prospective studies focusing on pancreatic ACC patients alone have been conducted to date, and most clinical studies investigating novel agents in pancreatic malignancy usually exclude pancreatic ACC. Therefore, more retrospective analyses of chemotherapy in patients with pancreatic ACC may help improve our understanding of this rare disease. Here, we present the clinical outcomes of patients with unresectable or metastatic pancreatic ACC who received chemotherapy in a tertiary referral cancer center.

\section{Materials and Methods}

We searched the clinical data warehouse of the Asan Medical Center (ABLE; Asan BiomedicaL rEsearch) and found 24 patients who had histologically documented pancreatic ACC with locally advanced unresectable, recurrent, or initially metastatic disease between January 1997 and March 2015. Among them, five patients were lost to follow-up after recurrence or refused chemotherapy and four patients were histologically diagnosed with mixed acinar-neuroendocrine carcinoma. Therefore, a total of 15 patients were included in the current analysis. We obtained clinical and pathological data from the review of patients' medical records. All radiological images were reviewed by the investigators.

Tumor responses were graded according to the Response Evaluation Criteria in Solid Tumor (RECIST) ver. 1.1 [9]. Progression-free survival (PFS) was calculated from the administration date for the first dose of chemotherapy to the date of disease progression or any cause of death, whichever occurred first. Overall survival (OS) was calculated from the first dose of chemotherapy to the date of death due to any cause. If patients were alive, they were censored at the time of last follow-up. Time to tumor progression (TTP) was esti- mated as the time between the start of chemotherapy and documented tumor progression. PFS and OS were estimated using the Kaplan-Meier method and compared by the logrank test.

To compare the activity of second-line regimens used, we analyzed the Growth Modulation Index (GMI) using the ratio of TTP at first-line chemotherapy (TTP1) to the TTP at second-line chemotherapy (TTP2) in patients who received second-line chemotherapy, similar to a previous study of another rare cancer [10]. The regimen with the higher GMI is considered to have better clinical efficacy. Considering only a small numbers of patients with pancreatic ACC were available to assess the efficacy of chemotherapy, this approach may be advantageous for measuring the relative activity among chemotherapeutic agents because patients serve as their own control. This generally increases statistical sensitivity because it eliminates the between-patient variability.

\section{Results}

\section{Clinical characteristics}

Baseline characteristics of the study population are summarized in Table 1. Overall, 13 patients received systemic chemotherapy, while two with locally advanced disease initially received concurrent chemoradiotherapy (CCRT) followed by sysftemic chemotherapy. The median age was 58 years (range, 29 to 72 years), and 13 patients (87\%) were male. Pancreatic head was the most common site of disease $(n=10,67 \%)$. Approximately half of the patients $(n=6,40 \%)$ had recurrent disease after curative resection and four (27\%) had locally advanced unresectable disease. The most common metastatic site was the liver $(\mathrm{n}=7,47 \%)$, followed by intra-abdominal lymph nodes $(\mathrm{n}=5,33 \%)$ and peritoneum $(\mathrm{n}=3,20 \%)$. Individual patient characteristics and their responses to treatment are summarized in S1 Table.

\section{Treatment and efficacy}

As first-line therapy, intravenous 5-fluorouracil (5-FU) was administered to four patients $(27 \%)$, gemcitabine to five $(33 \%)$, gemcitabine plus capecitabine (GEM-CAP) to two $(13 \%)$, oxaliplatin plus 5-FU/leucovorin (FOLFOX) to two $(13 \%)$, and CCRT followed by capecitabine maintenance therapy to two (13\%) (Table 2). In patients who received chemotherapy alone, partial response (PR) was achieved in three patients, indicating an overall response rate (ORR) of 23\%. Additionally, three patients with PR received infusional 
Table 1. Patient characteristics at baseline

\begin{tabular}{lc} 
Characteristic & No. $(\%)(\mathbf{n}=\mathbf{1 5})$ \\
\hline Age, median (range, yr) & $58(29-72)$ \\
Sex (male/female) & $13(87) / 2(13)$ \\
Primary tumor location & \\
$\quad$ Pancreatic head & $10(67)$ \\
Pancreatic body/ Tail & $5(33)$ \\
CA 19-9 (elevated) & $4(27)$ \\
Disease setting & \\
Recurrent & $6(40)$ \\
Locally advanced & $4(27)$ \\
Initially metastatic & $5(33)$ \\
Metastatic site & \\
Liver & $7(47)$ \\
Distant lymph nodes & $5(33)$ \\
Peritoneum & $3(20)$ \\
Others & $3(20)$ \\
Previous surgery in curative intent & $6(40)$ \\
Previous adjuvant chemotherapy (n=6) & $2(33)$ \\
\hline
\end{tabular}

A

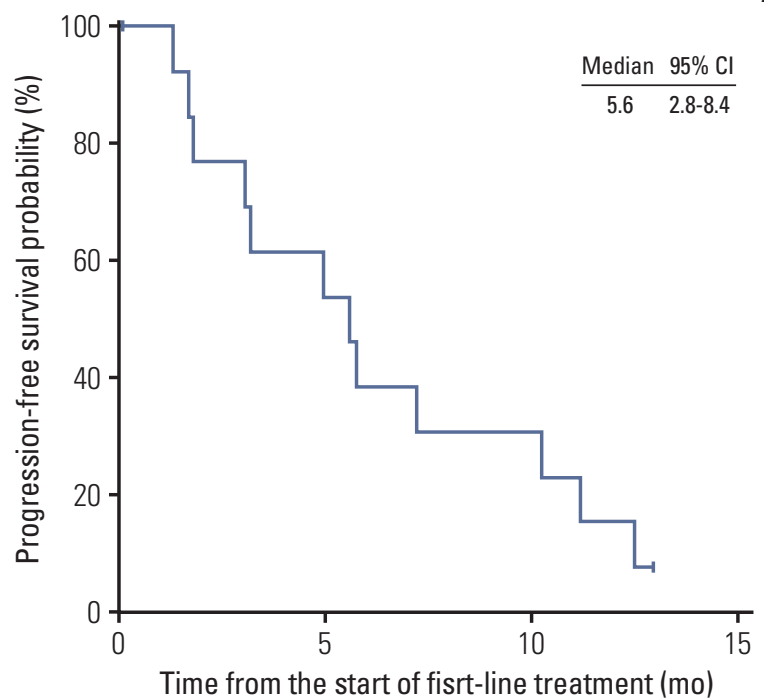

Table 2. First-line treatment and response

\begin{tabular}{ll} 
Variable & No. $(\boldsymbol{\%})(\mathbf{n}=\mathbf{1 5})$ \\
Treatment regimen & \\
Infusional 5-FU/Leucovorin & $4(27)$ \\
Gemcitabine monotherapy & $5(33)$ \\
GEM-CAP & $2(13)$ \\
FOLFOX & $2(13)$ \\
CCRT followed by capecitabine maintenance & $2(13)$ \\
Response to the first-line treatment & \\
CR $^{\text {a) }}$ & $1(7)$ \\
PR $^{\text {a) }}$ & $4(27)$ \\
SD & $5(33)$ \\
PD & $2(13)$ \\
NA & $3(20)$ \\
\hline
\end{tabular}

5-FU, 5-fluorouracil; GEM-CAP, gemcitabine plus capecitabine; FOLFOX, oxaliplatin plus 5-FU/leucovorin; CCRT, concurrent chemoradiotherapy; $\mathrm{CR}$, complete response; $\mathrm{PR}$, paritial response; $\mathrm{SD}$, stable disease; $\mathrm{PD}$, progressive disease; NA, not applicable; sLV5FU2, simplified leucovorin and 5-FU regimen. ${ }^{\mathrm{a})}$ One $\mathrm{CR}$ and one PR patients received CCRT with capecitabine followed by capecitabine for their locally advanced disease. The other three PR patients received sLV5FU2, GEM-CAP, and FOLFOX, b)Among three patients with NA for response evaluation, two patients were lost from early follow-up and one had no measurable lesion.

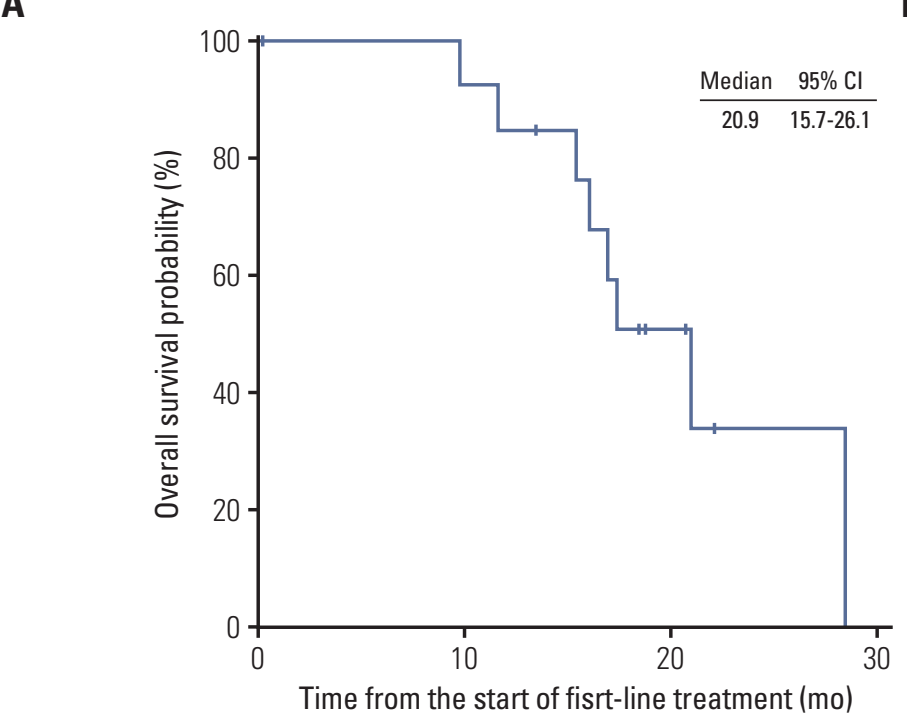

Fig. 1. Progression-free survival with first-line chemotherapy of patients with chemotherapy alone (A) and overall survival of all patients (B). CI, confidence interval. 
Table 3. Second-line chemotherapy and response

\begin{tabular}{ll} 
Variable & No. $(\%)(\mathrm{n}=8)$ \\
Chemotherapy regimen & \\
FOLFOX & $4(50)$ \\
Gemcitabine monotherapy & $4(50)$ \\
Response to the second-line chemotherapy & \\
CR & 0 \\
\hline PR & $3(37)$ \\
SD & $1(13)$ \\
PD & $4(50)$ \\
\hline
\end{tabular}

FOLFOX, oxaliplatin plus 5-fluorouracil/leucovorin; CR, complete response; $\mathrm{PR}$, partial response; $\mathrm{SD}$, stable disease; $\mathrm{PD}$, progressive disease. ${ }^{\text {a) }}$ All PR patients received FOLFOX.

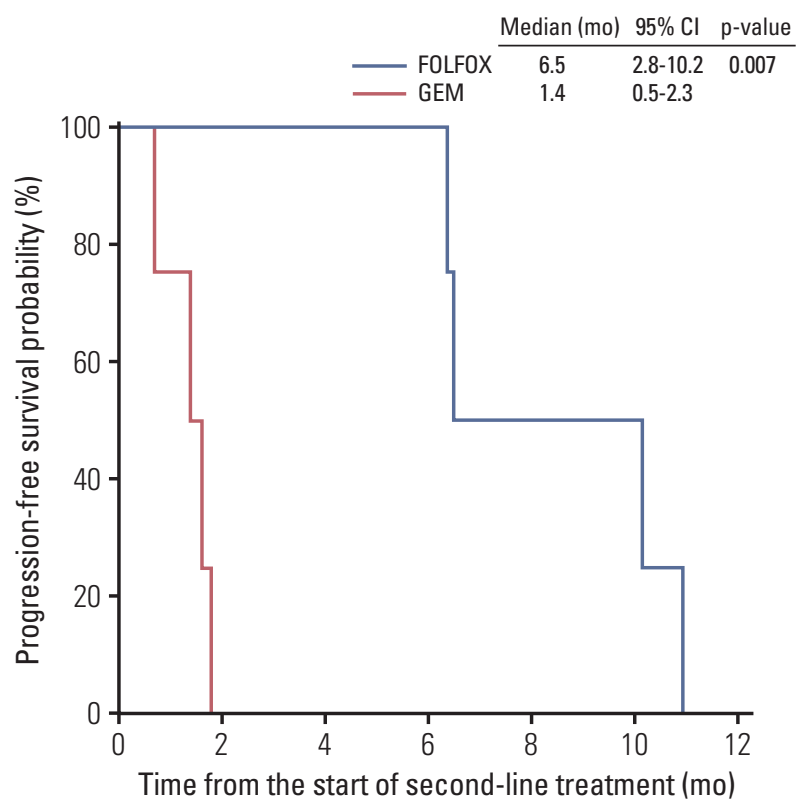

Fig. 2. Progression-free survival with second-line chemotherapy. CI, confidence interval; FOLFOX, oxaliplatin plus 5-fluorouracil/leucovorin; GEM, gemcitabine.
5-FU/leucovorin $(\mathrm{n}=1)$, GEM-CAP $(\mathrm{n}=1)$, and FOLFOX $(\mathrm{n}=1)$. Among two patients who received CCRT followed by capecitabine maintenance therapy for locally advanced disease, one patient achieved complete response and another one achieved PR. No patient treated with gemcitabine monotherapy achieved objective response (Table 2). The median PFS of patients with chemotherapy alone was 5.6 months (95\% confidence interval [CI], 2.8 to 8.4) (Fig. 1A). The median PFS was 11.2 months (95\% CI, 0.0 to 27.1) with intravenous 5-FU, 7.3 months with GEM-CAP, 5.6 months with FOLFOX, and 3.2 months (95\% CI, 3.0 to 3.4 ) with gemcitabine monotherapy. The median PFS of patients who received CCRT followed by capecitabine maintenance therapy was 14.5 months. Median OS for all patients was 20.9 months (95\% CI, 15.7 to 26.1) (Fig. 1B).

After disease progression while on first-line chemotherapy, second-line chemotherapy was administered to eight patients, with four receiving FOLFOX and four gemcitabine (Table 3). Objective response was achieved in three of the eight patients, indicating an ORR of $38 \%$. All three patients with PR received FOLFOX, and no patients who received gemcitabine achieved objective response (Table 3). Among the patients treated with second-line FOLFOX, gemcitabine monotherapy $(n=2)$, GEM-CAP $(n=1)$, and infusional 5-FU/leucovorin $(\mathrm{n}=1)$ had previously been administered. Patients treated with FOLFOX had significantly better PFS than those treated with gemcitabine monotherapy (median, 6.5 months; $95 \%$ CI, 2.8 to 10.2 vs. 1.4 months; $95 \%$ CI, 0.5 to 2.3; $\mathrm{p}=0.007$ ) (Fig. 2). The GMI was significantly higher in patients with FOLFOX (4.07; range, 0.87 to 8.30 ) than in those with gemcitabine ( 0.12 ; range, 0.08 to $0.25 ; \mathrm{p}=0.029)$ (Table 4$)$.

\section{Discussion}

In the current study, we retrospectively analyzed the clinical outcomes of patients with unresectable or metastatic pancreatic ACC. Our results suggest that oxaliplatin-contain-

Table 4. Comparison of the ratio of TTP1 to TTP2 in patients who received second-line chemotherapy

\begin{tabular}{lccc} 
Second-line chemotherapy & Gemcitabine alone & FOLFOX & p-value \\
TTP1, median (mo) & 5.8 & 1.7 & \\
TTP2, median (mo) & 1.4 & 6.5 & \\
GMI (TTP2/TTP1) & $0.12(0.08-0.25)$ & $4.07(0.87-8.30)$ & 0.029 \\
\hline
\end{tabular}

TTP1, time to progression at first-line chemotherapy; TTP2, time to progression at second-line chemotherapy; GMI, Growth Modulation Index. 
ing regimens may have better efficacy than gemcitabine monotherapy.

The baseline characteristics of our study population were similar to the results of previously published epidemiological studies in terms of age, sex, and tumor location [6,11-13]. Most patients were male ( $87 \%$ ) and the pancreatic head was the most common site of primary tumor $(67 \%)$. Consistent with the results of a previous retrospective study [14], the median OS in our patients was 20.9 months (95\% CI, 15.7 to 26.1). These results suggest that the overall prognosis of patients with unresectable or metastatic pancreatic ACC seems to be better than that of PDAC.

With first-line chemotherapy, the ORR was $23 \%$ and the median PFS was 5.6 months (95\% CI, 2.8 to 8.4). Monotherapy with intravenous 5-FU showed numerically longer PFS (median, 11.2 months) than other regimens, such as monotherapies with GEM-CAP (7.3 months), FOLFOX (5.6 months), and gemcitabine (3.2 months). The two patients with locally advanced disease who received upfront CCRT followed by capecitabine maintenance therapy showed the longest PFS (20.1 and 14.5 months).

In the second-line setting, FOLFOX showed better efficacy than gemcitabine monotherapy in terms of PFS and GMI (i.e., the ratio of TTP1 to TTP2). GMI was suggested as a potential end point of drug efficacy [15] and showed a strong relationship with survival outcome in pre-treated patients with sarcoma [16]. Patients administered FOLFOX had significantly better PFS than those administered gemcitabine monotherapy (median, 6.5 months; 95\% CI, 2.8 to 10.2 vs. 1.4 months; $95 \% \mathrm{CI}, 0.5$ to $2.3 ; \mathrm{p}=0.007$ ). GMI was also significantly higher in patients administered FOLFOX (4.07; range, 0.87 to 8.30 ) than in those administered gemcitabine monotherapy ( 0.12 ; range, 0.08 to $0.25 ; p=0.03$ ). Despite the large difference in terms of PFS between FOLFOX and gemcitabine, the number of patients in the second-line setting was too small to conclude whether FOLFOX was superior to gemcitabine, because of probable imbalance in baseline characteristics, including prognostic factors. Nevertheless, the significantly higher GMI with FOLFOX (4.07) than gemcitabine (0.12) suggests that oxaliplatin-containing regimens have better efficacy than gemcitabine, which has been the most popular regimen in pancreatic cancer to date. Indeed, a previous study conducted by the French Sarcoma Group found that a GMI $>1.33$ was highly associated with improved OS in the setting of second-line chemotherapy for patients with softtissue sarcoma [16]. In very rare types of cancer such as pancreatic ACC, GMI may be a good indicator to estimate the activity of agent through intra-patient comparison, which may decrease the issues related with confounding factors.

The promising efficacy of oxaliplatin-containing regimens in this study might be explained by the distinctive molecular characteristics of pancreatic ACC. A recent study showed that the molecular signature of ACC is different from that of PDAC. KRAS, TP53, CDKN2A (p16), and SMAD4 gene mutations were not typically found in pancreatic ACC, whereas the frequency of mutations in the adenomatous polyposis coli- $\beta$ catenin pathway, which is rarely detected in PDAC, was similar to those found in colorectal cancer $(7 \%-24 \%)$ [17-19]. These findings suggest that the chemotherapeutic approaches for ACC patients include agents known to have activity in colorectal cancer [14,20-22].

Improved efficacy with oxaliplatin in pancreatic ACC may be because of the frequent genomic alterations associated with inactivation of DNA repair genes. In preclinical studies, pancreatic tumors from $B R C A 2$ mutation carriers that showed evidence of loss of heterozygosity at the mutation site were associated with the development of ACC [23]. A recent Japanese study using whole-exome sequencing revealed that the loss of $B R C A 2$ expression was observed in $45 \%$ [24] of patients (5/11) with liver metastasis, one of whom achieved complete remission after cisplatin-based chemotherapy. Comprehensive genomic profiling of 44 pancreatic ACC also showed that approximately half of the pancreatic ACC patients $(45 \%)$ had inactivating genomic alterations in DNA repair genes (BRCA 1/2, ATM, MSH 1/2, RAD50, BRIP1, RANCA, and PALB2), and that BRCA2 mutations were detected in 20\% of pancreatic ACC [19]. Loss of function in DNA repair genes predisposes susceptibility to the platinum-based chemotherapy or poly(ADP-ribose) polymerase inhibitor; hence, the findings regarding DNA repair deficiencies in pancreatic ACC support our results with regard to the promising efficacy of oxaliplatin-containing regimens.

Multivariate analysis to exclude the impact of confounding factors could not be performed in this study because of the small number of patient included. Moreover, this study has inherent selection bias caused by its retrospective nature. Despite these limitations, this study has advantages in terms of a relatively large number of patients in the setting of unresectable or metastatic disease and that detailed information about the chemotherapeutic agents used was available.

\section{Conclusion}

In conclusion, our results suggest that the oxaliplatin-containing chemotherapy may have improved activity against pancreatic ACC compared with gemcitabine. This is supported by the results of recent studies demonstrating the distinctive genetic background of pancreatic ACC, including the high frequency of $B R C A$ mutations. We applied GMI during statistical analysis to overcome the limitations associated 
with the small populations and retrospective nature of our study. Nevertheless, it is still difficult to apply our results in general. Moreover, a large prospective multicenter trial is needed to address the rare incidence of pancreatic ACC.

Overall, recent findings, including those of the present study, indicate that chemotherapy strategies for unresectable or metastatic pancreatic ACC should be different from those for PDAC.

\section{Electronic Supplementary Material}

Supplementary materials are available at Cancer Research and Treatment website (http://www.e-crt.org).

\section{Conflicts of Interest}

Conflict of interest relevant to this article was not reported.

\section{Acknowledgments}

This study was supported by a grant (2015-0753) from the Asan Institute for Life Sciences, Asan Medical Center, Seoul, Korea.

\section{References}

1. Chen J, Baithun SI. Morphological study of 391 cases of exocrine pancreatic tumours with special reference to the classification of exocrine pancreatic carcinoma. J Pathol. 1985;146: 17-29.

2. Ordonez NG. Pancreatic acinar cell carcinoma. Adv Anat Pathol. 2001;8:144-59.

3. Seth AK, Argani P, Campbell KA, Cameron JL, Pawlik TM, Schulick RD, et al. Acinar cell carcinoma of the pancreas: an institutional series of resected patients and review of the current literature. J Gastrointest Surg. 2008;12:1061-7.

4. Mortenson MM, Katz MH, Tamm EP, Bhutani MS, Wang H, Evans DB, et al. Current diagnosis and management of unusual pancreatic tumors. Am J Surg. 2008;196:100-13.

5. Holen KD, Klimstra DS, Hummer A, Gonen M, Conlon K, Brennan $\mathrm{M}$, et al. Clinical characteristics and outcomes from an institutional series of acinar cell carcinoma of the pancreas and related tumors. J Clin Oncol. 2002;20:4673-8.

6. La Rosa S, Adsay V, Albarello L, Asioli S, Casnedi S, Franzi F, et al. Clinicopathologic study of 62 acinar cell carcinomas of the pancreas: insights into the morphology and immunophenotype and search for prognostic markers. Am J Surg Pathol. 2012;36:1782-95.

7. Klimstra DS, Heffess CS, Oertel JE, Rosai J. Acinar cell carcinoma of the pancreas: a clinicopathologic study of 28 cases. Am J Surg Pathol. 1992;16:815-37.

8. Butturini G, Pisano M, Scarpa A, D'Onofrio M, Auriemma A, Bassi C. Aggressive approach to acinar cell carcinoma of the pancreas: a single-institution experience and a literature review. Langenbecks Arch Surg. 2011;396:363-9.

9. Eisenhauer EA, Therasse P, Bogaerts J, Schwartz LH, Sargent D, Ford R, et al. New response evaluation criteria in solid tumours: revised RECIST guideline (version 1.1). Eur J Cancer.
2009;45:228-47.

10. Zalcberg JR, Verweij J, Casali PG, Le Cesne A, Reichardt P, Blay JY, et al. Outcome of patients with advanced gastrointestinal stromal tumours crossing over to a daily imatinib dose of $800 \mathrm{mg}$ after progression on $400 \mathrm{mg}$. Eur J Cancer. 2005;41:1751-7.

11. Wisnoski NC, Townsend CM Jr, Nealon WH, Freeman JL, Riall TS. 672 patients with acinar cell carcinoma of the pancreas: a population-based comparison to pancreatic adenocarcinoma. Surgery. 2008;144:141-8.

12. Schmidt CM, Matos JM, Bentrem DJ, Talamonti MS, Lillemoe KD, Bilimoria KY. Acinar cell carcinoma of the pancreas in the United States: prognostic factors and comparison to ductal adenocarcinoma. J Gastrointest Surg. 2008;12:2078-86.

13. Kitagami H, Kondo S, Hirano S, Kawakami H, Egawa S, Tanaka M. Acinar cell carcinoma of the pancreas: clinical analysis of 115 patients from Pancreatic Cancer Registry of Japan Pancreas Society. Pancreas. 2007;35:42-6.

14. Lowery MA, Klimstra DS, Shia J, Yu KH, Allen PJ, Brennan $\mathrm{MF}$, et al. Acinar cell carcinoma of the pancreas: new genetic and treatment insights into a rare malignancy. Oncologist. 2011;16:1714-20.

15. Von Hoff DD. There are no bad anticancer agents, only bad clinical trial designs: twenty-first Richard and Hinda Rosenthal Foundation Award Lecture. Clin Cancer Res. 1998;4: 1079-86.

16. Cousin S, Blay JY, Bertucci F, Isambert N, Italiano A, Bompas $\mathrm{E}$, et al. Correlation between overall survival and growth modulation index in pre-treated sarcoma patients: a study from the French Sarcoma Group. Ann Oncol. 2013;24:2681-5.

17. Abraham SC, Wu TT, Hruban RH, Lee JH, Yeo CJ, Conlon K, et al. Genetic and immunohistochemical analysis of pancreatic 
acinar cell carcinoma: frequent allelic loss on chromosome 11p and alterations in the APC/beta-catenin pathway. Am J Pathol. 2002;160:953-62.

18. Furlan D, Sahnane N, Bernasconi B, Frattini M, Tibiletti MG, Molinari F, et al. APC alterations are frequently involved in the pathogenesis of acinar cell carcinoma of the pancreas, mainly through gene loss and promoter hypermethylation. Virchows Arch. 2014;464:553-64.

19. Chmielecki J, Hutchinson KE, Frampton GM, Chalmers ZR, Johnson A, Shi C, et al. Comprehensive genomic profiling of pancreatic acinar cell carcinomas identifies recurrent RAF fusions and frequent inactivation of DNA repair genes. Cancer Discov. 2014;4:1398-405.

20. Simon M, Bioulac-Sage P, Trillaud H, Blanc JF. FOLFOX regimen in pancreatic acinar cell carcinoma: case report and review of the literature. Acta Oncol. 2012;51:403-5.

21. Pfrommer S, Weber A, Dutkowski P, Schafer NG, Mullhaupt
B, Bourquin JP, et al. Successful salvage chemotherapy with FOLFIRINOX for recurrent mixed acinar cell carcinoma and ductal adenocarcinoma of the pancreas in an adolescent patient. Case Rep Oncol. 2013;6:497-503.

22. Ang C, Herran LA, Lagunes DR, Klimstra DS, Kemeny NE. A case report of a patient with advanced acinar cell carcinoma of the pancreas: long-term survival with regional, systemic and targeted therapy. Tumori. 2013;99:e61-4.

23. Skoulidis F, Cassidy LD, Pisupati V, Jonasson JG, Bjarnason $\mathrm{H}$, Eyfjord JE, et al. Germline Brca2 heterozygosity promotes Kras(G12D)-driven carcinogenesis in a murine model of familial pancreatic cancer. Cancer Cell. 2010;18:499-509.

24. Furukawa T, Sakamoto H, Takeuchi S, Ameri M, Kuboki Y, Yamamoto T, et al. Whole exome sequencing reveals recurrent mutations in BRCA2 and FAT genes in acinar cell carcinomas of the pancreas. Sci Rep. 2015;5:8829. 